PROCEEDINGS OF THE

AMERICAN MATHEMATICAL SOCIETY

Volume 140, Number 7, July 2012, Pages 2479-2488

S 0002-9939(2011)11080-3

Article electronically published on October 27, 2011

\title{
FLAT PSEUDO-RIEMANNIAN HOMOGENEOUS SPACES WITH NON-ABELIAN HOLONOMY GROUP
}

\author{
OLIVER BAUES AND WOLFGANG GLOBKE
}

(Communicated by Jianguo Cao)

\begin{abstract}
We construct homogeneous flat pseudo-Riemannian manifolds with non-abelian fundamental group. In the compact case, all homogeneous flat pseudo-Riemannian manifolds are complete and have abelian linear holonomy group. To the contrary, we show that there do exist non-compact and non-complete examples, where the linear holonomy is non-abelian, starting in dimensions $\geq 8$, which is the lowest possible dimension. We also construct a complete flat pseudo-Riemannian homogeneous manifold of dimension 14 with non-abelian linear holonomy. Furthermore, we derive a criterion for the properness of the action of an affine transformation group with transitive centralizer.
\end{abstract}

\section{INTRODUCTION}

A flat pseudo-Riemannian manifold $M$ is called homogeneous if its isometry group acts transitively. As examples show [2, 4, non-compact flat pseudo-Riemannian homogeneous manifolds are not necessarily complete. The study of complete flat homogeneous pseudo-Riemannian manifolds was pioneered by Wolf in a series of papers [8, 9, 10]. Such manifolds are isometric to a manifold of the form $\mathbb{R}^{r, s} / \Gamma$, for some subgroup $\Gamma \leq \operatorname{Iso}\left(\mathbb{R}^{r, s}\right)$. Homogeneity implies that the centralizer of $\Gamma$ in Iso $\left(\mathbb{R}^{r, s}\right)$ acts transitively on $\mathbb{R}^{r, s}$. One basic observation is that in this situation the group $\Gamma$ is nilpotent of nilpotency class at most two. This fact also holds for the holonomy group $\Gamma$ of a non-complete homogeneous pseudo-Riemannian manifold. Apparently, it was believed for some time that $\Gamma$ or, which is considerably weaker, the linear part of $\Gamma$ should be abelian. However, as observed in [1, non-abelian fundamental groups $\Gamma$ appear for compact complete flat homogeneous pseudo-Riemannian manifolds.

In this paper, we present some additional new results on the structure of flat pseudo-Riemannian homogeneous manifolds. Although non-abelian fundamental groups $\Gamma$ do appear, in the compact case the linear holonomy is always abelian. In addition, we show that every homogeneous flat pseudo-Riemannian manifold of dimension less than eight has abelian linear holonomy.

As one of our main results, we give examples of homogeneous manifolds with nonabelian linear holonomy group. We construct an eight-dimensional non-complete manifold $U / \Gamma_{1}$, where $U$ is an open domain in $\mathbb{R}^{4,4}$, and a fourteen-dimensional complete manifold $\mathbb{R}^{7,7} / \Gamma_{2}$, both with non-abelian linear holonomy. The groups

Received by the editors October 1, 2010 and, in revised form, February 16, 2011.

2010 Mathematics Subject Classification. Primary 53C30, 57S30; Secondary 20 G05.

(C)2011 American Mathematical Society 2479

Reverts to public domain 28 years from publication 
$\Gamma_{1} \leq \operatorname{Iso}\left(\mathbb{R}^{4,4}\right)$ and $\Gamma_{2} \leq \operatorname{Iso}\left(\mathbb{R}^{7,7}\right)$ are isomorphic to the integral Heisenberg group on two generators and map injectively to their linear parts. These manifolds give the first examples of flat pseudo-Riemannian homogeneous manifolds with non-abelian linear holonomy group.

\section{Preliminaries}

Here, $\mathbb{R}^{r, s}$ denotes $\mathbb{R}^{r+s}$ endowed with a scalar product $\langle\cdot, \cdot\rangle$ of signature $(r, s)$, and Iso $\left(\mathbb{R}^{r, s}\right)$ denotes its group of isometries. Affine maps of $\mathbb{R}^{r+s}$ are written as $\gamma=(I+A, v)$, where $I+A$ is the linear part ( $I$ the identity matrix) and $v$ the translation part. Let im $A$ denote the image of $A$.

The groups $\Gamma \subset \operatorname{Iso}\left(\mathbb{R}^{r, s}\right)$ with transitive centralizer in $\operatorname{Iso}\left(\mathbb{R}^{r, s}\right)$ were studied first in [8]. We sum up some of the results for later reference. Note that all of the following holds also if the centralizer of $\Gamma$ is only required to have an open orbit in $\mathbb{R}^{r, s}$ (compare [1, Proposition 3.10] or [3, Lemma 4.1]).

Lemma 2.1. $\Gamma$ consists of affine transformations $\gamma=(I+A, v)$, where $A^{2}=0$, $v \perp \operatorname{im} A$ and $\operatorname{im} A$ is totally isotropic.

Lemma 2.2. For $\gamma_{i}=\left(I+A_{i}, v_{i}\right) \in \Gamma, i=1,2,3$, we have $A_{1} A_{2} v_{1}=0=A_{2} A_{1} v_{2}$, $A_{1} A_{2} A_{3}=0$ and $\left[\gamma_{1}, \gamma_{2}\right]=\left(I+2 A_{1} A_{2}, 2 A_{1} v_{2}\right)$.

Lemma 2.3. If $\gamma=(I+A, v) \in \Gamma$, then $\langle A x, y\rangle=-\langle x, A y\rangle, \operatorname{im} A=(\operatorname{ker} A)^{\perp}$, $\operatorname{ker} A=(\operatorname{im} A)^{\perp}$ and $A v=0$.

Theorem 2.4. $\Gamma$ is 2-step nilpotent (meaning $[\Gamma,[\Gamma, \Gamma]]=\{\operatorname{id}\}$ ).

For $\gamma=(I+A, v) \in \Gamma$, set $\operatorname{hol}(\gamma)=I+A$ (the linear component of $\gamma$ ). We write $A=\log (\operatorname{hol}(\gamma))$.

Definition 2.5. The linear holonomy group of $\Gamma$ is $\operatorname{hol}(\Gamma)=\{\operatorname{hol}(\gamma) \mid \gamma \in \Gamma\}$.

Let $x \in M$ and $\gamma \in \pi_{1}(M, x)$ be a loop. Then $\operatorname{hol}(\gamma)$ corresponds to the parallel transport $\Psi_{x}(\gamma): \mathrm{T}_{x} M \rightarrow \mathrm{T}_{x} M$ in a natural way; cf. [7, Lemma 3.4.4].

In the latest edition of the book [7, a characterization of those $\Gamma$ with abelian linear holonomy is given:

Proposition 2.6. The following are equivalent:

(1) $\operatorname{hol}(\Gamma)$ is abelian.

(2) If $\left(I+A_{1}, v_{1}\right),\left(I+A_{2}, v_{2}\right) \in \Gamma$, then $A_{1} A_{2}=0$.

(3) The space $U_{\Gamma}=\sum_{\gamma \in \Gamma} \operatorname{im} A$ is totally isotropic.

The proof of Proposition 2.6 uses only the lemmata above. Thus, the following structure theorem for groups $\Gamma$, such that the centralizer of $\Gamma$ has an open orbit in $\mathbb{R}^{r, s}$, and $\Gamma$ with abelian linear holonomy, holds:

Theorem 2.7. If $\mathrm{hol}(\Gamma)$ is abelian, then for every Witt basis with respect to $U_{\Gamma}$ (see section 4) and $(I+A, v) \in \Gamma$, the matrix $A$ is of the form

$$
A=\left(\begin{array}{lll}
0 & 0 & C \\
0 & 0 & 0 \\
0 & 0 & 0
\end{array}\right),
$$

where $C$ is a skew-symmetric $k \times k$ matrix $\left(k=\operatorname{dim} U_{\Gamma}\right)$. 
In section 3, we show that for compact $M$ the holonomy group hol $(\Gamma)$ is always abelian, and we present a more refined classification and structure theorem for the groups $\Gamma$ in the compact case. We give a modification for the structure theorem, Theorem 2.7, which holds for arbitrary $\Gamma$, in section 4. In section 5, we show that groups $\Gamma$ such that $\operatorname{hol}(\Gamma)$ is not abelian exist only for dimensions $\geq 8$, and in section 6 we give an example of such a group. This group does not act freely and therefore cannot be the fundamental group of a complete flat homogeneous pseudoRiemannian manifold $M$. But it gives rise to a non-complete example $M$. We also present an example of a group $\Gamma$ which acts freely on $\mathbb{R}^{7,7}$ and has a transitive centralizer. This group gives rise to a complete 14-dimensional homogeneous flat pseudo-Riemannian manifold with non-abelian linear holonomy group. To show that the groups $\Gamma$ involved act properly we derive in section 7 a criterion which shows that a discrete unipotent group acting freely on $\mathbb{R}^{n}$, and whose centralizer has an open orbit, acts properly on $\mathbb{R}^{n}$.

\section{Compact flat pseudo-Riemannian spaces}

In this section, let $M$ be a compact flat homogeneous pseudo-Riemannian manifold. By [5] (see [1, Corollary 4.5] for an alternative proof), $M$ must be complete. Therefore, $M=\mathbb{R}^{r, s} / \Gamma$, for some group $\Gamma \leq \operatorname{Iso}\left(\mathbb{R}^{r, s}\right)$ which acts properly discontinuously and freely on $\mathbb{R}^{r, s}$. Let $G$ be the centralizer of $\Gamma$ in $\operatorname{Iso}\left(\mathbb{R}^{r, s}\right)$. Since $M$ is homogeneous and compact, then, as follows from [1, Theorem 4.6], $G$ is a nilpotent Lie group which acts simply transitively on $\mathbb{R}^{r, s}$ by isometries. Let $x_{0} \in \mathbb{R}^{r, s}$ be a fixed basepoint. There is a unique left-invariant pseudo-Riemannian metric $\langle\cdot, \cdot\rangle_{G}$ on $G$ such that the orbit map $o: G \rightarrow \mathbb{R}^{r, s}, g \mapsto g \cdot x_{0}$, is an isometry. Moreover, the metric $\langle\cdot, \cdot\rangle_{G}$ is bi-invariant; see [1, Theorem 4.6]. The map $o$ induces an isometry $G / \tilde{\Gamma} \rightarrow M$, where $\tilde{\Gamma}$ is a lattice subgroup of $G$, which is isomorphic to $\Gamma$, and $G / \tilde{\Gamma}$ inherits the pseudo-Riemannian structure from $\left(G,\langle\cdot, \cdot\rangle_{G}\right)$. It also follows that $G$ is (at most) two-step nilpotent (see [1, Lemma 4.8]). Such manifolds $G / \tilde{\Gamma}$ necessarily have abelian linear holonomy group:

Theorem 3.1. Let $G$ be a Lie group with a bi-invariant flat pseudo-Riemannian metric $\langle\cdot, \cdot\rangle_{G}$, and $\tilde{\Gamma} \leq G$ be a lattice. Then the compact flat pseudo-Riemannian homogeneous manifold $G / \tilde{\Gamma}$ has abelian linear holonomy.

Proof. Let $\rho: G \rightarrow \operatorname{Iso}\left(\mathbb{R}^{r, s}\right)$ be the development representation of the rightmultiplication of $G$ and put $\Gamma=\rho(\tilde{\Gamma})$. Then, as above, there is an orbit map $o: G \rightarrow \mathbb{R}^{r, s}$, which is an isometry and satisfies $o(g \gamma)=\rho(\gamma) o(g)$ (cf. 1, Proposition 5.2]). This map induces an isometry $G / \tilde{\Gamma} \rightarrow \mathbb{R}^{r, s} / \Gamma$.

Let $\mathfrak{g}$ denote the Lie algebra of $G$. By [1, Proposition 3.3, Lemma 5.10], the differential of $\rho$ at the identity is equivalent to the affine representation $X \mapsto$ $\left(\frac{1}{2} \operatorname{ad}(X), X\right)$ of $\mathfrak{g}$ on the vector space of $\mathfrak{g}$. In particular, the linear part of the differential of $\rho$ is equivalent to the adjoint representation ad of $\mathfrak{g}$. Since $\mathfrak{g}$ is twostep nilpotent, the adjoint representation ad has abelian image. It follows that the linear part of $\rho(G)$ is abelian. Since $\Gamma \leq \rho(G)$, this implies that $\Gamma$ has abelian linear part. 
Remark 3.2. Let $\langle\cdot, \cdot\rangle_{\mathfrak{g}}$ denote the inner product induced on $\mathfrak{g}$ by $\langle\cdot, \cdot\rangle_{G}$. Biinvariance of $\langle\cdot, \cdot\rangle_{G}$ is equivalent to

$$
\langle[X, Y], Z\rangle_{\mathfrak{g}}=-\langle Y,[X, Z]\rangle_{\mathfrak{g}} .
$$

Identify $\mathfrak{g} \cong \mathbb{R}^{r, s}$ via the differential of $o$. Then $\gamma=(I+A, v)=\left(I+\frac{1}{2} \operatorname{ad}(X), X\right)$, where $X=\log (\gamma) \in \mathfrak{g}$. Therefore,

$$
U_{\Gamma}=\sum_{\gamma \in \Gamma} \operatorname{im} A=\sum_{X \in \log (\Gamma)} \operatorname{imad}(X)=[\mathfrak{g}, \mathfrak{g}]
$$

equals the commutator subalgebra of $\mathfrak{g}$. (The last equality holds because the elements in $\log (\Gamma)$ generate $\mathfrak{g}$, since $\Gamma$ is a lattice in $G$.) Using bi-invariance and 2-step nilpotency, it is easy to see that the space $U_{\Gamma}=[\mathfrak{g}, \mathfrak{g}]$ is totally isotropic. By Theorem 2.7, this is equivalent to $\operatorname{hol}(\Gamma)$ being abelian.

Corollary 3.3. Let $M=\mathbb{R}^{r, s} / \Gamma$ be a compact flat pseudo-Riemannian homogeneous manifold. Then hol $(\Gamma)$ is abelian.

To specify a bi-invariant pseudo-Riemannian metric on the Lie group $G$ it is equivalent to construct a bi-invariant inner product $\langle\cdot, \cdot\rangle_{\mathfrak{g}}$ on the Lie algebra of $G$. The metric is flat if and only if $\mathfrak{g}$ is two-step nilpotent. Below, we state a structure theorem for such pairs $\left(\mathfrak{g},\langle\cdot, \cdot\rangle_{\mathfrak{g}}\right)$, taken from [1, Theorem 5.15]. By the above, this yields a structure theorem for groups $\Gamma$ which are the fundamental groups of compact homogeneous flat pseudo-Riemannian manifolds.

Recall that for a Lie algebra $\mathfrak{a}$ and its dual $\mathfrak{a}^{*}$, a Lie product on the space $\mathfrak{a} \oplus \mathfrak{a}^{*}$ is given by

$$
\left[\left(X, X^{*}\right),\left(Y, Y^{*}\right)\right]=\left([X, Y], \operatorname{ad}^{*}(X) Y^{*}-\operatorname{ad}^{*}(Y) X^{*}+\omega(X, Y)\right),
$$

where $\mathrm{ad}^{*}$ denotes the coadjoint representation, and $\omega \in \mathrm{Z}^{2}\left(\mathfrak{a}, \mathfrak{a}^{*}\right)$ is a 2-cocycle for the adjoint representation. We use the notation $\mathfrak{g}=\mathfrak{a} \oplus_{\omega} \mathfrak{a}^{*}$ for this Lie algebra. An inner product of split signature on $\mathfrak{g}$ is defined by

$$
\left\langle\left(X, X^{*}\right),\left(Y, Y^{*}\right)\right\rangle_{\mathfrak{g}}=X^{*}(Y)+Y^{*}(X),
$$

and it can be shown to be bi-invariant if and only if the 3 -form $F_{\omega}\left(X_{1}, X_{2}, X_{3}\right)=$ $\left\langle\omega\left(X_{1}, X_{2}\right), X_{3}\right\rangle$ on $\mathfrak{a}$ is alternating. If $\mathfrak{a}$ is abelian, then $\mathfrak{a} \oplus_{\omega} \mathfrak{a}^{*}$ is a 2-step nilpotent Lie algebra with bi-invariant inner product $\langle\cdot, \cdot\rangle_{\mathfrak{g}}$.

Theorem 3.4. Let $\mathfrak{g}$ be a 2-step nilpotent Lie algebra with bi-invariant inner product $\langle\cdot, \cdot\rangle_{\mathfrak{g}}$. Then there exists an abelian Lie algebra $\mathfrak{a}$, an alternating 3 -form $F_{\omega}$ on $\mathfrak{a}$ and an abelian Lie algebra $\mathfrak{z}_{0}$ such that $\mathfrak{g}$ can be written as a direct product of metric Lie algebras

$$
\mathfrak{g}=\left(\mathfrak{a} \oplus_{\omega} \mathfrak{a}^{*}\right) \oplus \mathfrak{z}_{0}
$$

Proof. $[\mathfrak{g}, \mathfrak{g}]$ is an isotropic subspace of $\mathfrak{g}$. Bi-invariance shows that its orthogonal complement $[\mathfrak{g}, \mathfrak{g}]^{\perp}$ is the center $\mathfrak{z}(\mathfrak{g})$. Let $\mathfrak{a}$ denote the isotropic subspace dual to $[\mathfrak{g}, \mathfrak{g}]$ in $\mathfrak{g}$ (then $[\mathfrak{g}, \mathfrak{g}]$ can be identified with the dual space $\mathfrak{a}^{*}$ of $\mathfrak{a}$ ). Finally, let $\mathfrak{z}_{0}$ be a complement of $\mathfrak{a}^{*}$ in $\mathfrak{z}(\mathfrak{g})$, that is, $\mathfrak{z}(\mathfrak{g})=\mathfrak{a}^{*} \oplus \mathfrak{z}_{0}$. Then $\mathfrak{z}_{0}$ commutes with and is orthogonal to $\mathfrak{a}$ and $\mathfrak{a}^{*}$. So $\mathfrak{g}=\left(\mathfrak{a} \oplus_{\omega} \mathfrak{a}^{*}\right) \oplus \mathfrak{z}_{0}$ for some 2-cocycle $\omega \in Z^{2}\left(\mathfrak{a}, \mathfrak{a}^{*}\right)$. 


\section{Structure theorem}

Let $\Gamma \subset \operatorname{Iso}\left(\mathbb{R}^{r, s}\right)$ be such that its centralizer in $\operatorname{Iso}\left(\mathbb{R}^{r, s}\right)$ has an open orbit in $\mathbb{R}^{r, s}$. For short, we write $\Delta$ for the center of $\Gamma$. This group is abelian, so it satisfies the conditions of Theorem 2.7. We set $U_{\Gamma}=\sum_{\gamma \in \Gamma} \operatorname{im} A, U_{\Delta}=\sum_{\gamma \in \Delta} \operatorname{im} A$ and $U_{0}=U_{\Gamma} \cap U_{\Gamma}^{\perp}$, which is a totally isotropic subspace. It follows from Lemma 2.3 that

$$
U_{0}=U_{\Gamma} \cap U_{\Gamma}^{\perp}=\sum_{\gamma \in \Gamma} \operatorname{im} A \cap \bigcap_{\gamma \in \Gamma} \operatorname{ker} A .
$$

Lemma 4.1. $U_{\Delta} \perp U_{\Gamma}$.

Proof. Let $\gamma_{1}=\left(I+A_{1}, v_{1}\right) \in \Delta$ and $\gamma_{2}=\left(I+A_{2}, v_{2}\right) \in \Gamma$. As $\gamma_{1}$ is central, it follows from Lemma 2.2 that $A_{1} A_{2}=0$, so im $A_{2} \subset \operatorname{ker} A_{1}=\left(\operatorname{im} A_{1}\right)^{\perp}$ (see Lemma 2.3. . Hence $U_{\Gamma} \perp U_{\Delta}$.

It is easy to see that $U_{\Delta} \subseteq U_{\Gamma}, U_{\Delta} \subseteq U_{0}, U_{\Delta}^{\perp} \supseteq U_{0}^{\perp} \supseteq U_{\Gamma}^{\perp} \supseteq U_{0} \supseteq U_{\Delta}$.

Lemma 4.2. $A \cdot U_{\Delta}^{\perp} \subseteq U_{0}$ for all $(I+A, v) \in \Gamma$.

Proof. Let $y \in U_{\Delta}^{\perp}$. For all $x \in \mathbb{R}^{r, s}$ and $A, B \in \log (\operatorname{hol}(\Gamma))$,

$$
\langle B x, A y\rangle=-\langle A B x, y\rangle=0,
$$

by Lemma 2.3 and because $A B$ is central. Hence $A y \perp U_{\Gamma}$, that is, $A y \in U_{0}$.

The following proposition sums up the above:

Proposition 4.3. The chain of subspaces

$$
\mathbb{R}^{r, s} \supset U_{\Delta}^{\perp} \supset U_{0} \supset\{0\}
$$

is stabilized by $\log (\mathrm{hol}(\Gamma))$ such that each subspace is mapped to the next in the chain.

Given the totally isotropic subspace $U_{0}$, we can find a Witt basis for $\mathbb{R}^{r, s}$ with respect to $U_{0}$ as follows: If $k=\operatorname{dim} U_{0}$, there exists a basis for $\mathbb{R}^{r, s}$,

$$
\left\{u_{1}, \ldots, u_{k}, \quad w_{1}, \ldots, w_{n-2 k}, \quad u_{1}^{*}, \ldots, u_{k}^{*}\right\},
$$

such that $\left\{u_{1}, \ldots, u_{k}\right\}$ is a basis of $U_{0},\left\{w_{1}, \ldots, w_{n-2 k}\right\}$ is a basis of a non-degenerate subspace $W$ such that $U_{0}^{\perp}=U_{0} \oplus W$, and $\left\{u_{1}^{*}, \ldots, u_{k}^{*}\right\}$ is a basis of a space $U_{0}^{*}$ such that $\left\langle u_{i}, u_{j}^{*}\right\rangle=\delta_{i j}$ (then $U_{0}^{*}$ is called a dual space for $U_{0}$ ). Let $\tilde{I}$ denote the signature matrix representing the restriction of $\langle\cdot, \cdot\rangle$ to $W$ with respect to the chosen basis of $W$.

The following generalizes Theorem 2.7 ;

Theorem 4.4. Let $\gamma=(I+A, v) \in \Gamma$ and fix a Witt basis with respect to $U_{0}$. Then the matrix representation of $A$ in this basis is

$$
A=\left(\begin{array}{ccc}
0 & -B^{\top} \tilde{I} & C \\
0 & 0 & B \\
0 & 0 & 0
\end{array}\right),
$$

with $B \in \mathbb{R}^{(n-2 k) \times k}$ and $C \in \mathfrak{s o}_{k}$ (where $k=\operatorname{dim}_{\tilde{I}} U_{0}$ ). The columns of $B$ are isotropic and mutually orthogonal with respect to $\tilde{I}$. 
Proof. With respect to the given Witt basis, $A$ is represented by a matrix

$$
\left(\begin{array}{ccc}
A_{1} & -A_{2}^{\top} \tilde{I} & A_{3} \\
A_{4} & A_{5} & A_{2} \\
A_{6} & -A_{4}^{\top} \tilde{I} & -A_{1}^{\top}
\end{array}\right)
$$

with $A_{3}, A_{6}$ skew-symmetric, $A_{5} \in \mathfrak{s o}(\tilde{I})$. By Proposition 4.3, $A_{1}=0, A_{4}=0$, $A_{6}=0$, and also $A_{5}=0$. Set $B=A_{2}, C=A_{3}$.

The condition $A^{2}=0$ implies that $-B^{\top} \tilde{I} B=0$, so all columns of $B$ are isotropic and mutually orthogonal with respect to $\tilde{I}$.

\section{DIMENSION BOUNDS FOR NON-ABELIAN HOLONOMY GROUPS}

We sum up two rules which have to be satisfied by the representation matrices (4.3). Given matrices $A_{i}(i=1,2), B_{i}$ and $C_{i}$ refer to the respective matrix blocks in (4.3).

(1) Crossover rule: Given $A_{1}$ and $A_{2}$, let $b_{2}^{i}$ be a column of $B_{2}$ and $b_{1}^{k}$ a column of $B_{1}$. Then $\left\langle b_{1}^{k}, b_{2}^{i}\right\rangle=-\left\langle b_{1}^{i}, b_{2}^{k}\right\rangle$. In particular, $\left\langle b_{1}^{k}, b_{2}^{k}\right\rangle=0$, and $\left\langle b_{1}^{i}, b_{1}^{k}\right\rangle=0$. If $\left\langle b_{1}^{i}, b_{2}^{k}\right\rangle \neq 0$, then $b_{1}^{k}, b_{1}^{i}, b_{2}^{k}, b_{2}^{i}$ are linearly independent. (The product of $A_{1} A_{2}$ contains $-B_{1}^{\top} \tilde{I} B_{2}$ as the skew-symmetric upper right block, so its entries are the values $-\left\langle b_{1}^{k}, b_{2}^{i}\right\rangle$.)

(2) Duality rule: Assume $A_{1}$ is not central (that is, $A_{1} A_{2} \neq 0$ for some $A_{2}$ ). Then $B_{2}$ contains a column $b_{2}^{i}$ and $B_{1}$ a column $b_{1}^{j}$ such that $\left\langle b_{1}^{j}, b_{2}^{i}\right\rangle \neq 0$.

Theorem 5.1. Let $\Gamma \subset \operatorname{Iso}\left(\mathbb{R}^{r, s}\right)$ be a group acting on $\mathbb{R}^{n}, n=r+s$, whose centralizer in $\operatorname{Iso}\left(\mathbb{R}^{r, s}\right)$ has an open orbit. If $\mathrm{hol}(\Gamma)$ is non-abelian, then

$$
n \geq 8 \text {. }
$$

As Example 6.2 shows, this is a sharp lower bound.

Proof. If hol $(\Gamma)$ is not abelian, there exist $\gamma_{1}=\left(I+A_{1}, v_{1}\right), \gamma_{2}=\left(I+A_{2}, v_{2}\right)$ such that $A_{1} A_{2} \neq 0$ (Lemma 2.2).

Let $W$ be a vector space complement of $U_{0}$ in $U_{0}^{\perp}$, so $W$ is non-degenerate and $x \in \mathbb{R}^{r, s}$ can be written as $x=u+w+u^{*}$ with $u \in U_{0}, w \in W, u^{*} \in U_{0}^{*}$. Then

$$
A_{1} x=\left(\begin{array}{ccc}
0 & -B_{1}^{\top} \tilde{I} & C_{1} \\
0 & 0 & B_{1} \\
0 & 0 & 0
\end{array}\right) \cdot\left(\begin{array}{c}
u \\
w \\
u^{*}
\end{array}\right)=\left(\begin{array}{c}
-B_{1}^{\top} \tilde{I} w+C_{1} u^{*} \\
B_{1} u^{*} \\
0
\end{array}\right) .
$$

By the duality rule, there are columns in $B_{1}, B_{2}$ which are non-orthogonal to one another. Then, by the crossover rule, $B_{1}$ and $B_{2}$ together contain at least four linearly independent columns. This implies that $\operatorname{dim} W \geq 4$.

Further, $B_{1}^{\top} \tilde{I} B_{2} \neq 0$. So if $A_{3}=\left[A_{1}, A_{2}\right]$, this means that the skew-symmetric matrix $C_{3}$ is non-zero. Hence $C_{3}$ must have at least two columns, that is, $\operatorname{dim} U_{0} \geq$ 2. Then

$$
n=\operatorname{dim} U_{0}+\operatorname{dim} W+\operatorname{dim} U_{0}^{*} \geq 2+4+2=8
$$

holds.

Remark 5.2. With the additional assumption that the centralizer of $\Gamma$ in $\operatorname{Iso}\left(\mathbb{R}^{r, s}\right)$ acts transitively, the second author has a proof (to appear in his dissertation) that the dimension bound in Theorem 5.1 can be improved to $n \geq 14$. As Example 6.4 shows, this is a sharp lower bound. 


\section{EXAMPLES}

Lemma 6.1. If the centralizer of $\Gamma$ in $\operatorname{Iso}\left(\mathbb{R}^{r, s}\right)$ has an open orbit $U$, then the $\Gamma$-action preserves $U$, that is, $\Gamma . U=U$.

Proof. By taking the Zariski closure, we may assume from the beginning that $\Gamma$ is an algebraic subgroup of Iso $\left(\mathbb{R}^{r, s}\right)$. Since the elements of $\Gamma$ are unipotent, the algebraic group $\Gamma$ is also connected. The centralizer $G$ of $\Gamma$ is also an algebraic subgroup, and as such it has finitely many open orbits in $\mathbb{R}^{r, s}$ (cf. [1, Proposition 6.8]). The group $\Gamma$ permutes the open orbits of $G$. Since it is connected, $\Gamma$, in fact, preserves each orbit.

Example 6.2. Let $\Gamma_{4,4} \subset \mathrm{Iso}\left(\mathbb{R}^{4,4}\right)$ be the group generated by

$$
\gamma_{1}=\left(\left(\begin{array}{ccc}
I_{2} & -B_{1}^{\top} \tilde{I} & 0 \\
0 & I_{4} & B_{1} \\
0 & 0 & I_{2}
\end{array}\right),\left(\begin{array}{c}
0 \\
w_{1} \\
0
\end{array}\right)\right), \quad \gamma_{2}=\left(\left(\begin{array}{ccc}
I_{2} & -B_{2}^{\top} \tilde{I} & 0 \\
0 & I_{4} & B_{2} \\
0 & 0 & I_{2}
\end{array}\right),\left(\begin{array}{c}
0 \\
w_{2} \\
0
\end{array}\right)\right)
$$

in the basis representation (4.3). Here,

$$
B_{1}=\left(\begin{array}{cc}
-1 & 0 \\
0 & -1 \\
0 & -1 \\
-1 & 0
\end{array}\right), \quad w_{1}=\left(\begin{array}{l}
1 \\
0 \\
0 \\
1
\end{array}\right), \quad B_{2}=\left(\begin{array}{cc}
0 & -1 \\
1 & 0 \\
-1 & 0 \\
0 & 1
\end{array}\right), \quad w_{2}=\left(\begin{array}{c}
0 \\
-1 \\
1 \\
0
\end{array}\right)
$$

and $\tilde{I}=\left(\begin{array}{cc}I_{2} & 0 \\ 0 & -I_{2}\end{array}\right)$ is the signature matrix of $W$. Their commutator is

$$
\gamma_{3}=\left[\gamma_{1}, \gamma_{2}\right]=\left(\left(\begin{array}{ccc}
I_{2} & 0 & C_{3} \\
0 & I_{4} & 0 \\
0 & 0 & I_{2}
\end{array}\right),\left(\begin{array}{c}
u_{3} \\
0 \\
0
\end{array}\right)\right)
$$

with

$$
C_{3}=\left(\begin{array}{cc}
0 & -4 \\
4 & 0
\end{array}\right), \quad u_{3}=\left(\begin{array}{c}
0 \\
-4
\end{array}\right) .
$$

One checks that $A_{i}^{2}=0$ and that $\gamma_{3}$ commutes with $\gamma_{1}, \gamma_{2}$. Therefore, $\Gamma_{4,4}$ is isomorphic to the discrete Heisenberg group on two generators.

In the chosen basis, the pseudo-scalar product is represented by the matrix

$$
Q=\left(\begin{array}{ccc}
0 & 0 & I_{2} \\
0 & \tilde{I} & 0 \\
I_{2} & 0 & 0
\end{array}\right)
$$

The following elements $S \in \mathfrak{i s o}\left(\mathbb{R}^{4,4}\right)$, where $\mathfrak{i s o}\left(\mathbb{R}^{4,4}\right)$ denotes the Lie algebra of Iso $\left(\mathbb{R}^{r, s}\right)$, commute with $\left(A_{1}, v_{1}\right)$ and $\left(A_{2}, v_{2}\right)$ :

$$
S=\left(\left(\begin{array}{ccc}
S_{1} & -S_{2}^{\top} \tilde{I} & 0 \\
0 & S_{3} & S_{2} \\
0 & 0 & -S_{1}^{\top}
\end{array}\right),\left(\begin{array}{l}
x \\
y \\
z
\end{array}\right)\right)
$$

where $x=\left(x_{1}, x_{2}\right)^{\top}, y=\left(y_{1}, y_{2}, y_{3}, y_{4}\right)^{\top}, z=\left(z_{1}, z_{2}\right)^{\top}$ are arbitrary and

$$
S_{1}=\left(\begin{array}{cc}
z_{1} & z_{2} \\
z_{2} & -z_{1}
\end{array}\right), \quad S_{2}=\left(\begin{array}{cc}
-y_{1} & y_{3}-y_{2} \\
-y_{2} & y_{1}+y_{4} \\
-y_{3} & 0 \\
-y_{4} & 0
\end{array}\right), \quad S_{3}=\left(\begin{array}{cccc}
0 & 0 & -z_{2} & -z_{1} \\
0 & 0 & z_{1} & -z_{2} \\
-z_{2} & z_{1} & 0 & 0 \\
-z_{1} & -z_{2} & 0 & 0
\end{array}\right) .
$$


Hence the elements $\exp (S)$ are contained in the centralizer of $\Gamma_{4,4}$ in $\operatorname{Iso}\left(\mathbb{R}^{r, s}\right)$. As $x, y, z$ are arbitrary, the centralizer of $\Gamma_{4,4}$ has an open orbit $U$ through the point 0 . The set of all elements $S$ is not a Lie subalgebra of the centralizer.

Corollary 6.3. There exists a flat incomplete homogeneous pseudo-Riemannian manifold of signature $(4,4)$ with non-abelian linear holonomy group.

Proof. By Lemma 6.1 and Proposition 7.3. $\Gamma_{4,4}$ acts properly discontinuously and freely on every open orbit $U$ of its centralizer in $\operatorname{Iso}\left(\mathbb{R}^{4,4}\right)$. So $M_{4,4}=U / \Gamma_{4,4}$ is a homogeneous manifold. The unit vector $e_{7}$ is a fixed point for $\gamma_{3} \in \Gamma_{4,4}$, so the action of the centralizer is not transitive. Hence, $U \neq \mathbb{R}^{4,4}$, and $M$ is incomplete.

Example 6.4. Let $\Gamma_{7,7} \subset \operatorname{Iso}\left(\mathbb{R}^{7,7}\right)$ be the group generated by

$$
\gamma_{1}=\left(\left(\begin{array}{ccc}
I_{5} & -B_{1}^{\top} \tilde{I} & C_{1} \\
0 & I_{4} & B_{1} \\
0 & 0 & I_{5}
\end{array}\right),\left(\begin{array}{c}
0 \\
0 \\
u_{1}^{*}
\end{array}\right)\right), \quad \gamma_{2}=\left(\left(\begin{array}{ccc}
I_{5} & -B_{2}^{\top} \tilde{I} & C_{2} \\
0 & I_{4} & B_{2} \\
0 & 0 & I_{5}
\end{array}\right),\left(\begin{array}{c}
0 \\
0 \\
u_{2}^{*}
\end{array}\right)\right)
$$

in the basis representation (4.3). Here,

$$
\begin{gathered}
B_{1}=\left(\begin{array}{ccccc}
-1 & 0 & 0 & 0 & 0 \\
0 & -1 & 0 & 0 & 0 \\
0 & -1 & 0 & 0 & 0 \\
-1 & 0 & 0 & 0 & 0
\end{array}\right), \quad C_{1}=\left(\begin{array}{ccccc}
0 & 0 & 0 & 0 & 0 \\
0 & 0 & 0 & 0 & 0 \\
0 & 0 & 0 & 0 & -1 \\
0 & 0 & 0 & 0 & 0 \\
0 & 0 & 1 & 0 & 0
\end{array}\right), \quad u_{1}^{*}=\left(\begin{array}{c}
0 \\
0 \\
0 \\
-1 \\
0
\end{array}\right), \\
B_{2}=\left(\begin{array}{ccccc}
0 & -1 & 0 & 0 & 0 \\
1 & 0 & 0 & 0 & 0 \\
-1 & 0 & 0 & 0 & 0 \\
0 & 1 & 0 & 0 & 0
\end{array}\right), \quad C_{2}=\left(\begin{array}{ccccc}
0 & 0 & 0 & 0 & 0 \\
0 & 0 & 0 & 0 & 0 \\
0 & 0 & 0 & 0 & 0 \\
0 & 0 & 0 & 0 & -1 \\
0 & 0 & 0 & 1 & 0
\end{array}\right), \quad u_{2}^{*}=\left(\begin{array}{c}
0 \\
0 \\
1 \\
0 \\
0
\end{array}\right),
\end{gathered}
$$

and $\tilde{I}=\left(\begin{array}{cc}I_{2} & 0 \\ 0 & -I_{2}\end{array}\right)$ is the signature matrix of $W$. Their commutator is

$$
\gamma_{3}=\left[\gamma_{1}, \gamma_{2}\right]=\left(\left(\begin{array}{ccc}
I_{5} & 0 & C_{3} \\
0 & I_{4} & 0 \\
0 & 0 & I_{5}
\end{array}\right),\left(\begin{array}{c}
u_{3} \\
0 \\
0
\end{array}\right)\right)
$$

with

$$
C_{3}=\left(\begin{array}{ccccc}
0 & -4 & 0 & 0 & 0 \\
4 & 0 & 0 & 0 & 0 \\
0 & 0 & 0 & 0 & 0 \\
0 & 0 & 0 & 0 & 0 \\
0 & 0 & 0 & 0 & 0
\end{array}\right), \quad u_{3}=\left(\begin{array}{l}
0 \\
0 \\
0 \\
0 \\
2
\end{array}\right)
$$

One checks that $A_{i}^{2}=0$ and that $\Gamma_{7,7}$ is isomorphic to a discrete Heisenberg group.

In the chosen basis, the pseudo-scalar product is represented by the matrix

$$
Q=\left(\begin{array}{ccc}
0 & 0 & I_{5} \\
0 & \tilde{I} & 0 \\
I_{5} & 0 & 0
\end{array}\right)
$$

The following elements $S \in \mathfrak{i s o}\left(\mathbb{R}^{7,7}\right)$ commute with $\left(A_{1}, v_{1}\right)$ and $\left(A_{2}, v_{2}\right)$ :

$$
S=\left(\left(\begin{array}{ccc}
S_{1} & -S_{2}^{\top} \tilde{I} & S_{3} \\
0 & 0 & S_{2} \\
0 & 0 & -S_{1}^{\top}
\end{array}\right),\left(\begin{array}{l}
x \\
y \\
z
\end{array}\right)\right)
$$


where $x=\left(x_{1}, \ldots, x_{5}\right)^{\top}, y=\left(y_{1}, \ldots, y_{4}\right)^{\top}, z=\left(z_{1}, \ldots, z_{5}\right)^{\top}$ are arbitrary and

$$
\begin{gathered}
S_{1}=\left(\begin{array}{ccccc}
0 & 0 & 0 & 0 & -2 z_{2} \\
0 & 0 & 0 & 0 & 2 z_{1} \\
0 & 0 & 0 & 0 & 0 \\
0 & 0 & 0 & 0 & 0 \\
0 & 0 & 0 & 0 & 0
\end{array}\right), \quad S_{2}=\left(\begin{array}{ccccc}
0 & 0 & -z_{2} & z_{1} & 0 \\
0 & 0 & z_{1} & z_{2} & 0 \\
0 & 0 & -z_{1} & z_{2} & 0 \\
0 & 0 & z_{2} & z_{1} & 0
\end{array}\right), \\
S_{3}=\left(\begin{array}{cccccc}
0 & 0 & -y_{2}-y_{3} & y_{4}-y_{1} & 0 \\
0 & 0 & y_{1}+y_{4} & y_{3}-y_{2} & 0 \\
y_{2}+y_{3} & -y_{1}-y_{4} & 0 & z_{5} & -z_{4} \\
y_{1}-y_{4} & y_{2}-y_{3} & -z_{5} & 0 & z_{3} \\
0 & 0 & z_{4} & -z_{3} & 0
\end{array}\right) .
\end{gathered}
$$

The linear part of such a matrix $S$ is conjugate to a strictly upper triangular matrix via conjugation with the matrix

$$
T=\left(e_{1}, e_{2}, e_{3}, e_{4}, e_{7}+e_{8}, e_{5}, e_{6}, e_{9}, e_{10}, e_{11}, e_{12}, e_{13}, e_{14}, e_{7}-e_{8}\right),
$$

where $e_{i}$ denotes the $i$ th unit vector. Hence, the elements $\exp (S)$ generate a unipotent group of isometries whose translation parts contain all of $\mathbb{R}^{14}$. Therefore, the centralizer of $\Gamma_{7,7}$ in Iso $\left(\mathbb{R}^{7,7}\right.$ ) acts transitively (see [1, Corollary 6.27] or [3, Theorem 4.2]). In particular, $\Gamma_{7,7}$ acts freely on $\mathbb{R}^{7,7}$.

It can be verified that the set of all matrices $S$ forms a 3 -step nilpotent Lie subalgebra of the centralizer algebra. Hence the set of all $\exp (S)$ forms a unipotent group of isometries acting simply transitively on $\mathbb{R}^{7,7}$.

Corollary 6.5. There exists a flat complete homogeneous pseudo-Riemannian manifold of signature $(7,7)$ with non-abelian linear holonomy group.

Proof. By Proposition 7.2 , the group $\Gamma_{7,7}$ acts properly discontinuously and freely on $\mathbb{R}^{7,7}$. So $M=\mathbb{R}^{7,7} / \Gamma_{7,7}$ is a complete homogeneous manifold.

\section{Properness of aCtions With transitive CEnTRAlizer}

Recall that an action of a Lie group $L$ on a locally compact Hausdorff space $X$ is called proper if and only if for all compact sets $K \subset X$ the set $\{\ell \in L \mid \ell K \cap K \neq \emptyset\}$ is compact.

Lemma 7.1. Let $X=G / H$ be a homogeneous space, where $G$ is a Lie group and $H$ is a closed subgroup. Let $L \leq \operatorname{Diff}(X)$ be a group of diffeomorphisms of $X$ which centralizes $G$. Then $L$ acts properly on $X$ if and only if $L$ is a closed subgroup of $\operatorname{Diff}(X)$ with respect to the compact open topology.

Proof. Choose a basepoint $x_{0} \in X$ such that $H=G_{x_{0}}$ is the stabilizer of $x_{0}$. Then $X$ is homeomorphic to $G / H$ via the orbit map $o: G / H \rightarrow X, g \mapsto g \cdot x_{0}$. The right-action of $\mathrm{N}_{G}(H)$ on $G$ induces a continuous homomorphism onto the centralizer $\mathrm{Z}_{X}(G)$ of $G$ in $\operatorname{Diff}(X)$. Let $\bar{L}$ denote the preimage of $L$ in $\mathrm{N}_{G}(H)$. In particular, if $L$ is closed in $\operatorname{Diff}(X)$, then $\bar{L}$ is closed in $G$. Note that $X / L=G / \bar{L}$ is a Hausdorff space if and only if the subgroup $\bar{L}$ is closed in $G$. Since $L$ acts freely on $X, X / L$ is Hausdorff if and only if $L$ acts properly on $X$. This proves the lemma. 
We can apply this criterion in the affine situation, as follows:

Proposition 7.2. Let $L \leq \operatorname{Aff}\left(\mathbb{R}^{n}\right)$ be a subgroup whose centralizer in $\operatorname{Aff}\left(\mathbb{R}^{n}\right)$ acts transitively on $\mathbb{R}^{n}$. Then the action of $L$ on $\mathbb{R}^{n}$ is proper if and only if $L$ is a closed subgroup of $\mathrm{Aff}\left(\mathbb{R}^{n}\right)$.

Similarly, assume that the centralizer $G$ of $L$ in $\operatorname{Aff}\left(\mathbb{R}^{n}\right)$ has an open orbit $U=G \cdot x_{0}$ which is preserved by $L$. Then $L$ acts freely on $U$, and the action is proper if and only if $L$ is closed in $\operatorname{Diff}(U)$. Since $\operatorname{Diff}(U) \cap \operatorname{Aff}\left(\mathbb{R}^{n}\right)$ is closed in $\operatorname{Aff}\left(\mathbb{R}^{n}\right)$ (cf. [1, Lemma 6.9]), the above proposition generalizes to:

Proposition 7.3. Let $L \leq \operatorname{Aff}\left(\mathbb{R}^{n}\right)$ be a subgroup whose centralizer in $\operatorname{Aff}\left(\mathbb{R}^{n}\right)$ acts transitively on an open subset $U$ of $\mathbb{R}^{n}$. If $L$ preserves $U$, then the action of $L$ on $U$ is proper if and only if $L$ is a closed subgroup of $\operatorname{Aff}\left(\mathbb{R}^{n}\right)$.

Remark 7.4. Püttmann [6, Section 4.2] gives an example of a free action of the abelian group $\left(\mathbb{C}^{2},+\right)$ on $\mathbb{C}^{5}$ by unipotent affine transformations such that the quotient is not a Hausdorff space. Hence the action is not proper.

\section{ACKNowledgement}

The authors wish to thank Joseph A. Wolf for reading the first draft of this article and for his continuing interest in the subject.

\section{REFERENCES}

[1] O. Baues, Flat Pseudo-Riemannian manifolds and prehomogeneous affine representations, in 'Handbook of Pseudo-Riemannian Geometry and Supersymmetry', EMS, IRMA Lect. Math. Theor. Phys. 16, 2010, pp. 731-817. MR2681607

[2] D. Duncan, E. Ihrig, Incomplete flat homogeneous geometries, Differential geometry: Geometry in mathematical physics and related topics (Los Angeles, CA, 1990), 197-202, Proc. Sympos. Pure Math., 54, Part 2, Amer. Math. Soc., Providence, RI, 1993. MR 1216539 (94f:53115)

[3] D. Duncan, E. Ihrig, Flat pseudo-Riemannian manifolds with a nilpotent transitive group of isometries, Ann. Global Anal. Geom. 10 (1992), no. 1, 87-101. MR1172623 (93f:53055)

[4] D. Duncan, E. Ihrig, Homogeneous spacetimes of zero curvature, Proc. Amer. Math. Soc. 107 (1989), no. 3, 785-795. MR975639 (90b:53075)

[5] J. Marsden, On completeness of homogeneous pseudo-riemannian manifolds, Indiana Univ. Math. J. 22 (1972/73), 1065-1066. MR0319128 (47:7674)

[6] A. Püttmann, Free affine actions of unipotent groups on $\mathbb{C}^{n}$, Transform. Groups 12 (2007), no. 1, 137-151. MR2308033 (2008g:14121)

[7] J.A. Wolf, Spaces of constant curvature, 6th edition, Amer. Math. Soc., 2011. MR 2742530

[8] J.A. Wolf, Homogeneous manifolds of zero curvature, Trans. Amer. Math. Soc. 104 (1962), 462-469. MR.0140050 (25:3474)

[9] J.A. Wolf, Flat homogeneous pseudo-Riemannian manifolds, Geom. Dedicata 57 (1995), no. 1, 111-120. MR1344776 (96f:53070)

[10] J.A. Wolf, Isoclinic spheres and flat homogeneous pseudo-Riemannian manifolds, Crystallographic groups and their generalizations (Kortrijk, 1999), 303-310, Contemp. Math., 262, Amer. Math. Soc., Providence, RI, 2000. MR 1796139 (2002c:53118)

Department of Mathematics, Institute for Algebra and Geometry, Karlsruhe Institute of Technology, 76128 Karlsruhe, Germany

E-mail address: baues@kit.edu

Department of Mathematics, Institute for Algebra and Geometry, Karlsruhe Institute of Technology, 76128 Karlsruhe, Germany

E-mail address: globke@math.uni-karlsruhe.de 